\section{BIOINFORMATION \\ Discovery at the interface of physical and biological sciences}

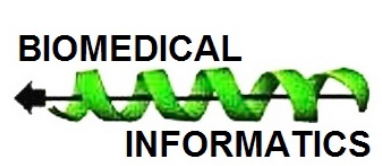

WWw.bioinformation.net

Volume 12(3)

\title{
Insights using the molecular model of Lipoxygenase from Finger millet (Eleusine coracana (L.))
}

\section{Hypothesis}

\author{
Apoorv Tiwari1,2,\#, Himanshu Avashthi1,2,\#, Richa Jha5,\#, Ambuj Srivastava,,\#, Vijay Kumar Garg1,2,\#, \\ Pramod Wasudev Ramteke, ${ }^{3, \#}$ and Anil Kumar1,"\#
}

${ }^{1}$ Department of Molecular Biology \& Genetic Engineering, CBSH, G. B. Pant University of Agriculture \& Technology, Pantnagar-263145, Uttarakhand, Bharat (India). ${ }^{2}$ Department of Computational Biology \& Bioinformatics, JSBB, Sam Higginbottom Institute of Agriculture, Technology and Sciences, Allahabad-211007, Uttar Pradesh, Bharat (India). ${ }^{3}$ Department of Biological Science, School of Basic Science, Sam Higginbottom Institute of Agriculture, Technology and Sciences, Allahabad-211007, Uttar Pradesh, Bharat (India). 4Department of Biotechnology, Indian Institute of Technology Madras, Chennai-600036, Tamil Nadu, Bharat (India). 5 Uttaranchal Institute of Technology, Uttaranchal University, Arcadia Grant, Dehradun-248007, Uttarakhand Bharat (India). Pramod Wasudev Ramteke - E-mail: pwramteke@yahoo.com; Prof. Anil Kumar - Email: anilkumar.mbge@gmail.com; *Corresponding author; \#Equal contribution

Received January 30, 2016; Revised May 17, 2016; Accepted May 18, 2016; Published June 15, 2016

\begin{abstract}
Lipoxygenase-1 (LOX-1) protein provides defense against pests and pathogens and its presence have been positively correlated with plant resistance against pathogens. Linoleate is a known substrate of lipoxygenase and it induces necrosis leading to the accumulation of isoflavonoid phytoalexins in plant leaves. Therefore, it is of interest to study the structural features of LOX-1 from Finger millet. However, the structure ofLOX-1 from Finger millet is not yet known. A homology model of LOX-1 from Finger millet is described. Domain architecture study suggested the presence of two domains namely PLAT (Phospho Lipid Acyl Transferase) and lipoxygenase. Molecular docking models of linoleate with lipoxygenase from finger millet, rice and sorghum are reported. The features of docked models showed that finger millet have higher pathogen resistance in comparison to other cereal crops. This data is useful for the molecular cloning of fulllength LOX-1 gene for validating its role in improving plant defense against pathogen infection and for various other biological processes.
\end{abstract}

Keywords: Lipoxygenase, Eleusine coracana, pest resistance, homology modeling, Structure prediction, docking, protein-protein interaction.

Abbreviations: LOX_Lipoxygenase; RMSD_Root mean square deviation; JA_Jasmonic acid; GRAVY_Grand average hydropathy.

\section{Background:}

Lipoxygenases (Fatty-acid metabolism) are a class of ironcontaining and most widely studied enzymes that catalyze the hydroperoxidation of polyunsaturated fatty acids (PUFAs) in lipids containing a cis, cis-1, 4-pentadiene structure. These are widely found in more than 60 species of plant and animal kingdom. This enzyme plays a significant role in formation of secondary metabolites in sweet basil [1]. LOXs producing hydroperoxy fatty acids, highly reactive compounds that may be toxic and can initiate lipid peroxidation, causes damage to various cell components. Furthermore, hydroperoxides can be metabolized into volatile aldehydes and jasmonates and plays vital role in defense machinery as well as various physiological processes in plant systems [2].

Finger millet (Eleusine coracana) is one of the important and probably very primitive millets in India. In all the cereals, this has highest amount of calcium and potassium. In comparison to white 


\section{BIOINFORMATION \\ Diseovery at the interface of physical and biological sciences}

\section{Open access}

rice, finger millet has higher dietary fiber, minerals and sulfur containing amino acids [2]. Due to the presence of plant oxidative enzymes such as lioxygenases, peroxidases, ascorbate and polyphenol oxidases of finger millet showing higher abilities of insect pest resistance [3]. LOX catalyze steps in the Jasmonic acid (JA) biosynthetic pathway to produce JA in response to plant defenses against insects [4]. LOX also play an important role in host resistance against the corn earworm Helicoverpa zea. It is also presents in soybean and red bean leguminous hosts plants of Helicoverpa zea [5]. LOX play potential role in plant defence as well as host-pathogen interaction in tomato. The rate of substrate conversion was about nine times superior with linoleic and linolenic acid in compare to arachidonic acid [6].

Lipoxygenase is one of the largest monomeric families with iron and non-sulphur properties [7]. LOX isoenzyme is almost uniformly present in the plant kingdom and it takes part in different physiological processes including flowering, seed germination, growth, development, pest resistance, and senescence or response to wound [2]. These proteins perform their functions by protein-protein interaction and post translational modifications [8]. The lipoxygenase pathway becomes an initial and key step in the interaction of plants with insects, or biotic and abiotic stresses at distinct stages of development [9]. After mechanical wounding or insect feeding, the induction of LOX transcript in the same plant has been observed in several species. It was clearly established for a normal wound response in Arabidopsis and potato there would be necessity of chloroplast isoform [10].
Earlier X-ray diffraction method was used to determine the three dimensional structure of lipoxygenase- 1 in soybean [11]. This threedimensional structure of lipoxygenase- 1 shows $38.0 \%$ of helical content and $13.9 \%$ of beta-sheet content [12]. LOX-1 is closely related to C-type lectins [13]. Crystal structure of LOX-1 ligand binding domain, along with a short stalk region connecting domain to the membrane-spanning region, linked by an inter-chain disulfide bond as a homodimer [14]. The biochemical function of protein is directly linked with its three dimensional structure. In earlier scientific era it was believed that the structure of a protein determines its function, however, with revolution in computational biology, functions are also been used to solve structure of a protein. To understand biochemical function and interaction properties of a protein at molecular level, three dimensional spatial arrangements is foremost required. However, the number of available protein sequences surpasses the available three dimensional protein structures. In order to overcome this, homology modeling approach is used. Various computational methods were applied to unravel information hidden inside the amino acid sequence and their structure.

In the present investigation, in silico analysis and homology modeling was used to characterize lipoxygenase protein in Finger millet. This method is much cost and time-effective compared to wet experimental techniques like X-ray crystallography and NMR.

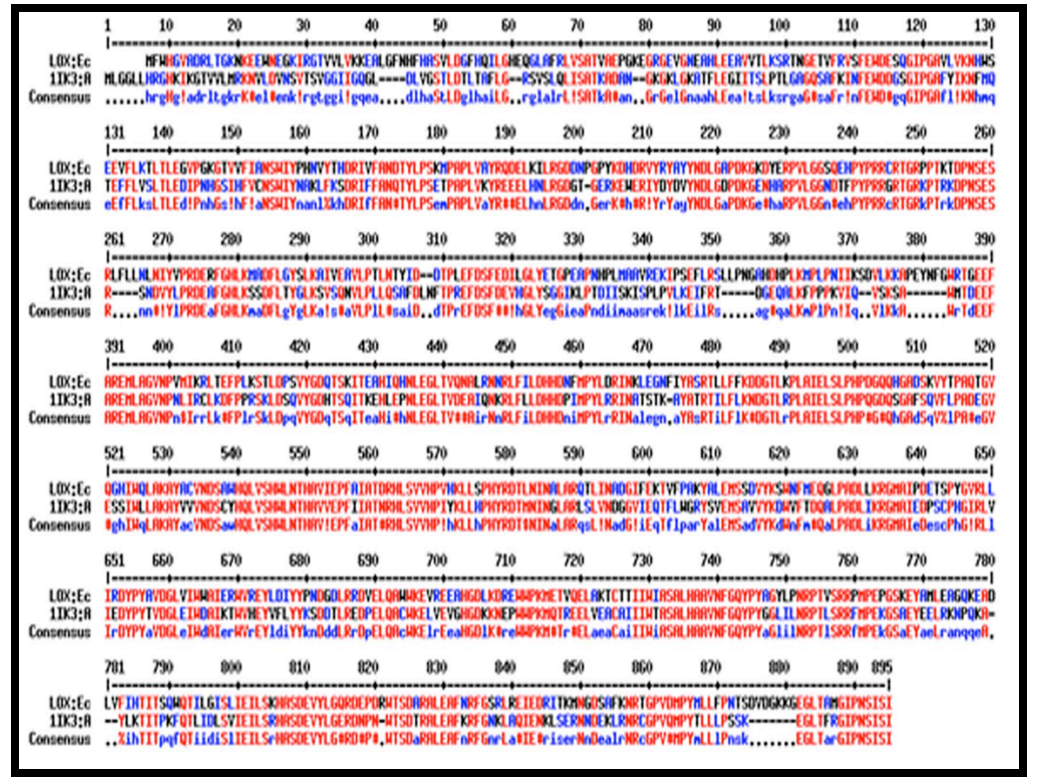

Figure 1: Target LOX (Eleusine coracana) and template sequence (1IK3: Chain A) were aligned using ESpript3.0 program by utilizing the clustalW algorithm. Similar residues are shown in blue and black colour, identical amino acids are shown in red colour. 


\section{Open access}

\section{Methodology:}

\section{Retrieval of target Sequence}

The protein sequence of lipoxygenase; Eleusine coracana [Accession: AHH24969, GI: 639542363 and length: 887 amino acid] was retrieved in FASTA format from National Centre for Biotechnology Information (NCBI) [15].

\section{Template Selection and Model Building}

The protein sequence of lipoxygenase was subjected to heuristic pairwise alignment search program BLASTp against the PDB database to identify suitable template(s) for 3D structure modeling. To select the template, phylogenetic analysis was also done by using ClustalW2 and tree was generated using MEGA6 software. On the basis of BLAStp analysis we have constructed the 3Dimensional model of target lipoxygenase protein of finger millet using homology modeling algorithm by Modeller 9.12 [16].

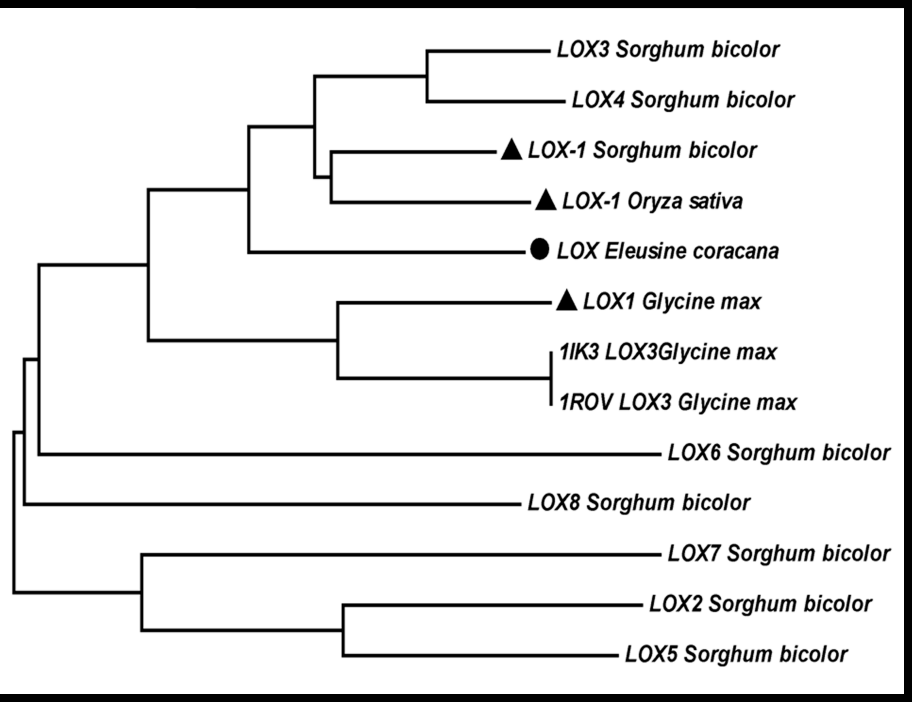

Figure 2: Phylogenetic tree of lipoxygenase proteins of sorghum, soybean and finger millet. Maximum-likelihood tree was generated using MEGA6 software using full length protein sequences. LOX finger millet (Eleusine coracana) target sequence showing close relations with LOX-1 of rice (Oryza sativa) sorghum (Sorghum bicolor) and soybean (Glycine max) as indicated in dark filled triangle shape.

\section{Model validation, visualization and evaluation}

Energy minimization was performed with the partial implementation of the GROMOS96 force-field with 200 iterations of steepest descent using SPDB viewer (http://spdbv.vital-it.ch/). To validate and identify errors in the predicted model of LOX, modeled structures were submitted to the SAVES: Structural Analysis and Verification Server [17]. Different modules of SAVES server like Procheck [18] was used to analyze stereo-chemical properties of protein structures using Ramachandran Plot [19], Verify3D [20] to assign 3D-1D score and ERRAT to check the quality factor, ProSA [21] was used for the analysis of Z-score and energy plot. The three dimensional structures of modeled LOX protein were visualized by PyMOL v1.3 Viewer [22] as shown in Figure 4. Root Mean Square Deviation (RMSD) analysis was done using UCSF Chimera [23] between target and template structure to see how much modeled protein structure deviates from the template structure.

\section{Sub-cellular localization prediction}

Subcellular localization of lipoxygenase protein was predicted using CELLO: Subcellular Localization Prediction System [24]. We have selected the location with the highest score, since it is the most likely.

\section{Physico-chemical characterization}

Physico-chemical properties such as theoretical pI (isoelectric point), molecular weight, $-R$ and $+R$ (total number of positively and negatively charged residues), II (instability index) [25], AI (aliphatic index), GRAVY (grand average of hydropathicity) [26] of all residues were calculated using MultiFASTA ProtParam interface tool [27].

\section{Functional characterization}

SMART database was used to identify the domain in lipoxygenase protein, which was then used by CYS_REC program [28] to locate disulphide bridges "SS bond" between cysteine residues. This tool provides the positions and total number of cysteine residues etc.

\section{Submission of modeled protein in protein model database (PMDB)} The modeled structure of Lipoxygenase protein was successfully submitted in PMDB: Protein model database [26], generated by modeller software without any stereo-chemical mistakes. The submitted model can be accessed via its PMDB ID [PM0079607].

\section{Active site prediction}

After completion of modeling and refinement of the LOX-1 structure, it was submitted to Computed Atlas of Surface Topography of proteins (CASTp) server for prediction of the possible binding sites [29]. Five active binding sites were predicted in the target protein structure and binding site having greater volume of each protein were selected for further investigations.

\section{Molecular Docking}

The docking of lipoxygenase-1 was performed with linoleate molecule (CID: 4573899) retrieved from ChemSpider database having 279.438 Dalton Average mass, 2 hydrogen bond acceptors and 1 hydrogen bond donor using AutoDock Vina [30]. AutoDock Vina requires three dimensional structure of both protein and ligand molecule. It performs rigid docking where the protein and ligand molecule are non-flexible, so the optimal geometry of the

\section{BIOMEDICAL}




\section{BIOINFORMATION \\ Discovery at the interface of physical and biological sciences}

\section{Open access}

ligand molecule is determined by docking approach. Python 2.5 and MGLTools from Molecular Graphics Laboratory were also used to perform docking. The best conformation and lowest energy were selected from the AutoDock Vina results.

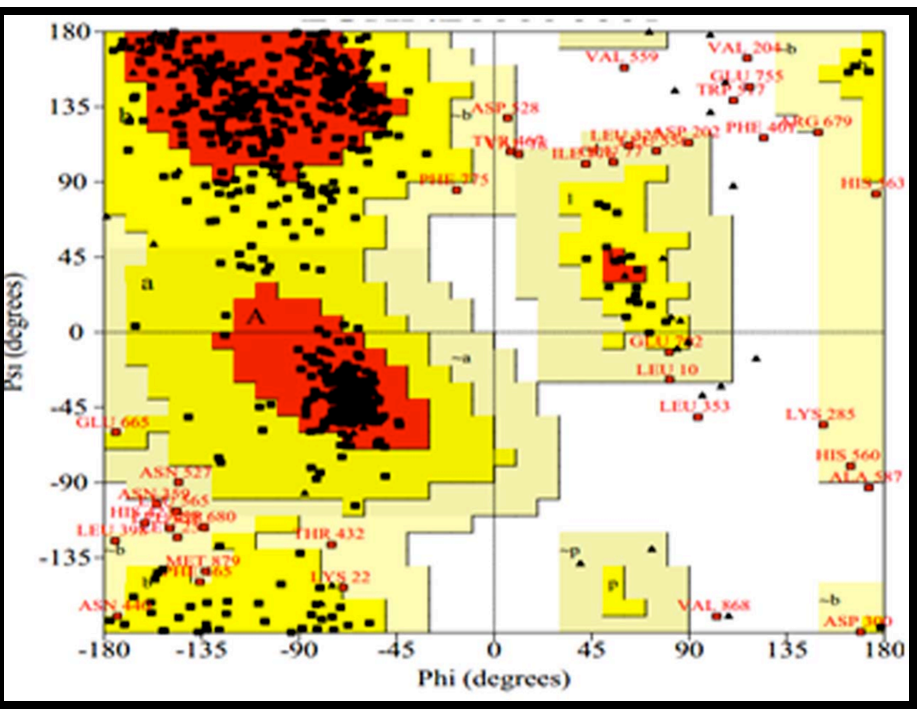

Figure 3: Ramachandran plot of lipoxygenase (model 1) in finger millet validated by PROCHECK program. $87.1 \%$ residues in most favoured region; 9.1\% residues in additional allowed region; $2.3 \%$ residues in generously allowed region; $0.7 \%$ residues in disallowed region.

\section{Protein- Protein interaction network prediction}

Functional protein association network was predicted using STRING database version 10 [3]. This database provides a decisive evaluation of protein-protein interactions, including direct as well as indirect associations.

\section{Results \& Discussion:}

In order to derive the 3D structure of lipoxygenase protein in finger millet, homology-modeling approach was used due to unavailability of experimental structure of this versatile protein.

\section{Phylogenetic analysis for template selection}

Lipoxygenase finger millet was taken as query sequence to perform local alignment search (Blastp) against protein data bank (PDB) for searching the homologous sequence (template) and family belongingness. According to BLAST result lipoxygenase- 3 of soybean [Glycine max] complex with 13(s)-hydroperoxy-9(z), 11(e)octadecadienoic acid PDBID: 1IK3 (Chain A) shown higher sequence identity $(54 \%)$ in comparison to LOX-3 treated with Cumene Hydroperoxide soybean [Glycine max] PDBID: 1ROV (Chain A) 53\% sequence identity. Based on high sequence identity, lower E-value (0.0) and maximum score (992), template (PDBID:
1IK3) was selected. It was further validated by phylogenetic analysis, to compare lipoxygenase protein sequences of finger millet, LOX1, LOX2, LOX3, LOX4, LOX5, LOX6, LOX7, LOX8 of sorghum, LOX1 of rice and template structure sequences were taken into account for multiple sequence alignment using ClustalW2 program, henceforth phylogenetic tree was generated using MEGA6 software. LOX finger millet was found very similar to LOX1 rice and sorghum as shown in results (Table 1) with $64.48 \%$ and $64.42 \%$ sequence identity, which was also displayed in phylogenetic tree. LOX finger millet is also highly conserved with 1IK3 template structure of soybean with $54.03 \%$ identity as shown in Figure 1. These results illustrate close relation of the LOX in finger millet with LOX-1 proteins. Hence, it can be conclude that query sequence from finger millet is LOX-1 protein. The phylogenetic tree also shows close relatedness of LOX of finger millet with LOX-1 of soybean, as shown in Figure 2. Therefore, the LOX-1 of soybean protein structure should be used to model LOX protein of finger millet, however because of unavailability of structure of LOX-1 protein in PDB, we have chosen LOX-3 protein of soybean (1IK3) for building 3D model.

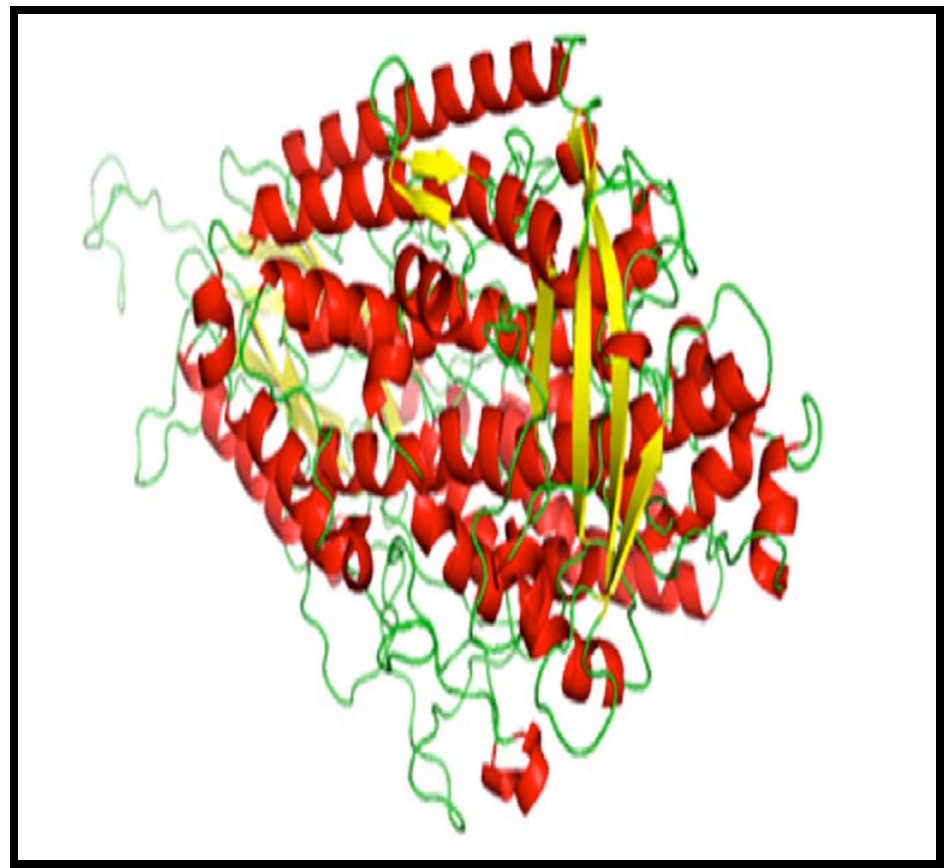

Figure 4: Ribbon diagram for modeled structure of lipoxygenase protein, visualized using PyMOL v1.3 Viewer. alpha-helix, betastand and turns is represented in red, yellow and green colors, respectively. 


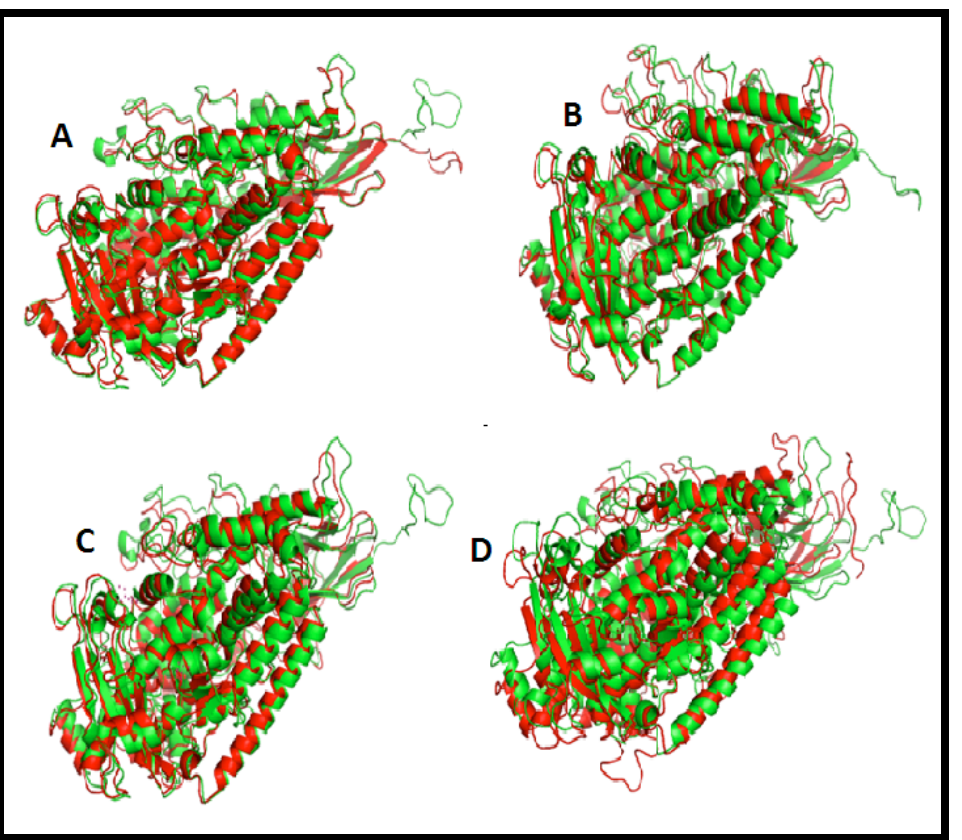

Figure 5: Superimpostion of structures (A) Finger millet (green colour) and rice (red colour) with $0.298 \AA$ RMSD value. (B) Rice (green colour) and 1IK3 template structure (red colour) with $0.391 \AA$ RMSD value. (C) Finger millet (green colour) and 1IK3 template structure (red colour) with $0.496 \AA$ RMSD value. (D) Finger millet (green colour) and sorghum (red colour) with 0.263 A RMSD value. That indicate the Sorghum show much structural similarity with Finger millet rather than Rice.

\section{Model Assessment and Refinement PROCHECK analysis}

All predicted model of lipoxygenase protein was compared with Modeller 9.12 and on the basis of most favoured, additional allowed, generously allowed and disallowed region, the best result was shown by model number 1 . The PROCHECK analysis provides an idea of the stereochemical quality and also overall assessment of protein through Ramachandran plot, as shown in Figure 3. For best predicted model, total $674(87.9 \%)$ residues were found in most favoured, $70(9.1 \%)$ in additional allowed region, $18(2.3 \%)$ in generously allowed region and $5(0.7 \%)$ in disallowed region, which ensure the good quality of predicted model, along with this overall $-0.18 \mathrm{G}$-factor was found for lipoxygenase, which suggests that the modeled structure might exhibit acceptable qualities.

\section{ERRAT and VERIFY 3D analysis}

Structure verification servers such as Verify 3D and ERRAT were used to validate modeled structure. Compatibility of an atomic model with its own amino acid sequence was determined using VERIFY 3D and found good compatibility with $95.38 \%$ score.
Statistics of non-bonded interactions was analyzed using ERRAT shown overall quality factor 62.073 this implies that structure is errorless.

\section{ProSA analysis}

The z-score for lipoxygenase (model 1) protein was observed to be within the range of score for native proteins of similar size, which indicate a good quality model with the z-score (-11.92).

\section{Prediction of subcellular localization}

Different proteins are located at different cellular compartment to acquire different function, however, sometimes it is difficult to experimentally determine. On the basis of score the most common localizations are: cytoplasm, mitochondrial, chloroplast, peroxisomal, nuclear, extracellular, endoplasmic reticulum, plasma membrane, golgi bodies, lysosomal, skeletal and vacuole with $3.609,0.374,0.239,0.218,0.152,0.148,0.080,0.070,0.063,0.024,0.014$ and 0.008 score. Hence, it could be speculated that lipoxygenase protein located mainly in cytoplasm and have very less chance to locate in vacuole. Similar study has been done on soybean lipoxygenase [31], and found that lipoxygenase involved in nitrogen storage and partitioning and located in cytosol.

\section{Characterization of physico-chemical properties}

The physico-chemical parameters viz., molecular weight, theoretical isoelectric point (Ip), total number of positively and negatively charged residues, instability index, aliphatic index and grand average hydropathy (GRAVY) were computed using the Expasy's ProtParam tool. Calculated isoelectric point value for lipoxygenase (model 1) is 5.90 which is less than normal value (7.00), indicating their acidic nature. LOX protein has significantly low and high, instability index $<40$ and aliphatic index (84.55), respectively, which suggest that this protein should be stable for wide range of temperature. The very low GRAVY index (-0.426) of LOX protein infers that protein has hydrophilic character for better interaction with water [25].

\section{Structural and functional analysis}

Two domains Polycystin-1, Lipoxygenase, Alpha-Toxin (PLAT) or Lipoxygenase homology (LH2) and lipoxygenase domain were found in LOX-1 finger millet which starts from residue number 21 and 175 and ends with 166 and 864 respectively. Same domains were also identified in LOX-1 rice which starts from 15 and 174 and ends with 159 and 844 respectively. This indicates that LOX finger millet and rice is highly structurally similar, but the positions of these domains are different from rice.. Therefore we belive that the function of LOX-1 finger millet is might be similar to rice LOX-1. Over expression of PLAT conferred increased abiotic stress tolerance, including cold, drought and salt stress [32]. 


\section{BIOINFORMATION}

Discovery at the interface of physical and biological sciences

\section{Open access}

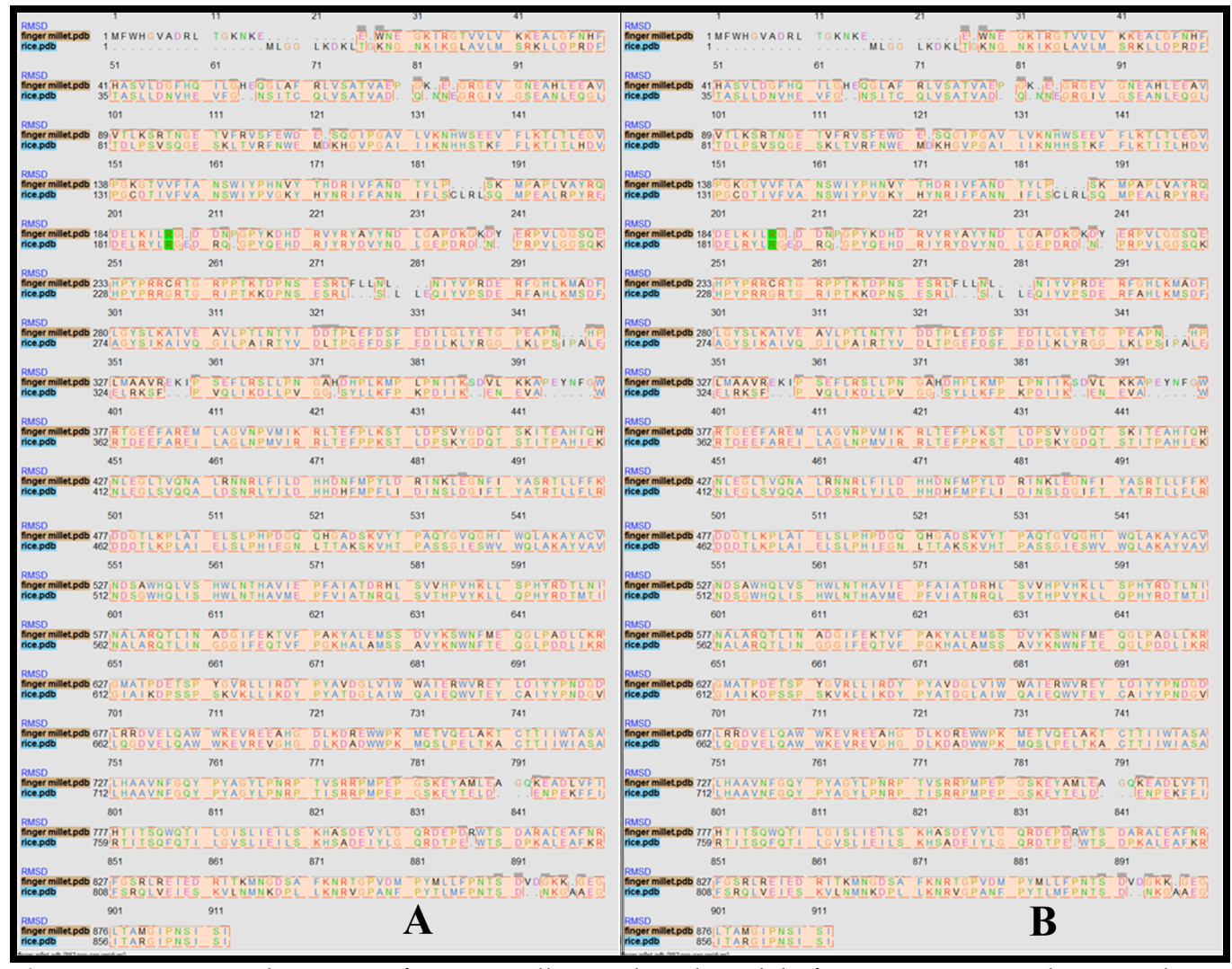

Figure 6: Structure alignment of Finger millet predicted model of Lipoxygenase with rice and sotghum. The first line denotes the secondary structure of Finger millet Lipoxygenase protein and the second line shows the number and position of identical residues clustering shown in (A) Structure-structure alignment between finger millet and rice lipoxygenase with $62.96 \%$ identity (B) finger millet and sorghum lipoxygenase with $31.47 \%$ identity.

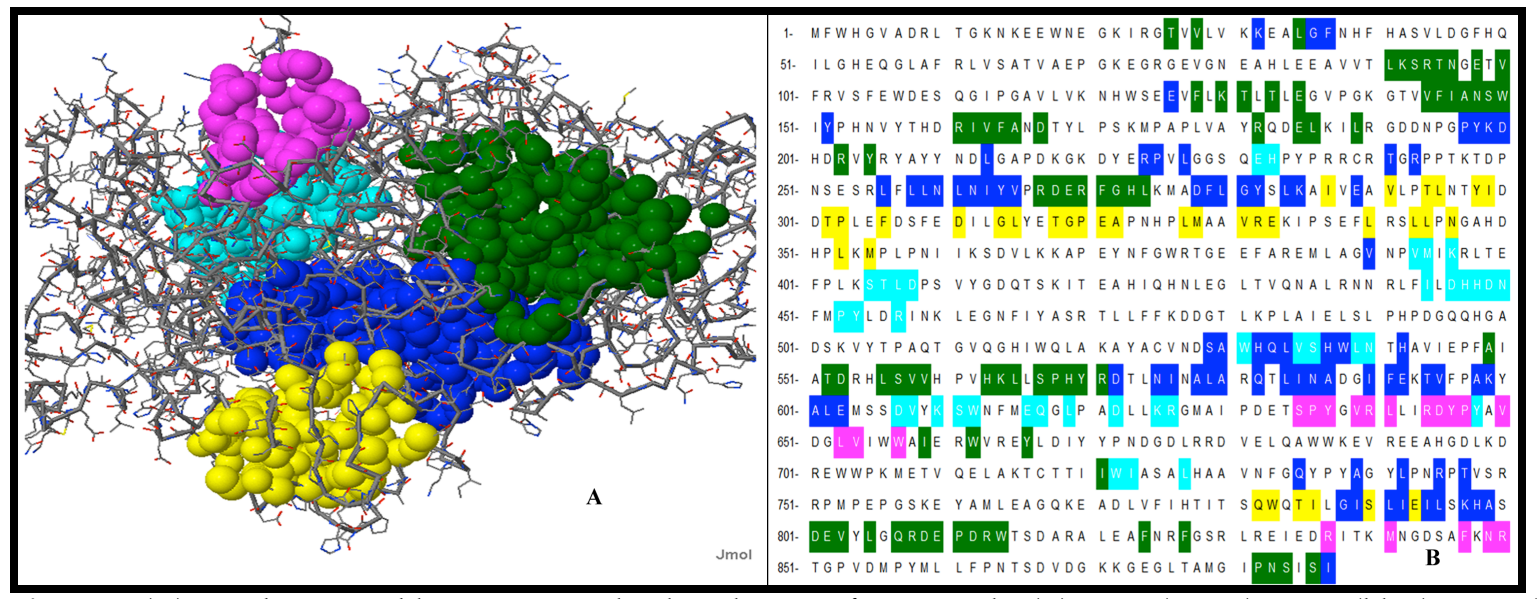

Figure 7: (A) Five best possible active sites displayed in wireframe mode; (B) site 1 (green), site 2 (blue), site 3 (cyan), site 4 (yellow) and site 5 (pink) shown in the predicted 3D model.

ISSN 0973-2063 (online) 0973-8894 (print) 


\section{BIOINFORMATION \\ Discovery at the interface of physical and biological sciences}

\section{Open access}

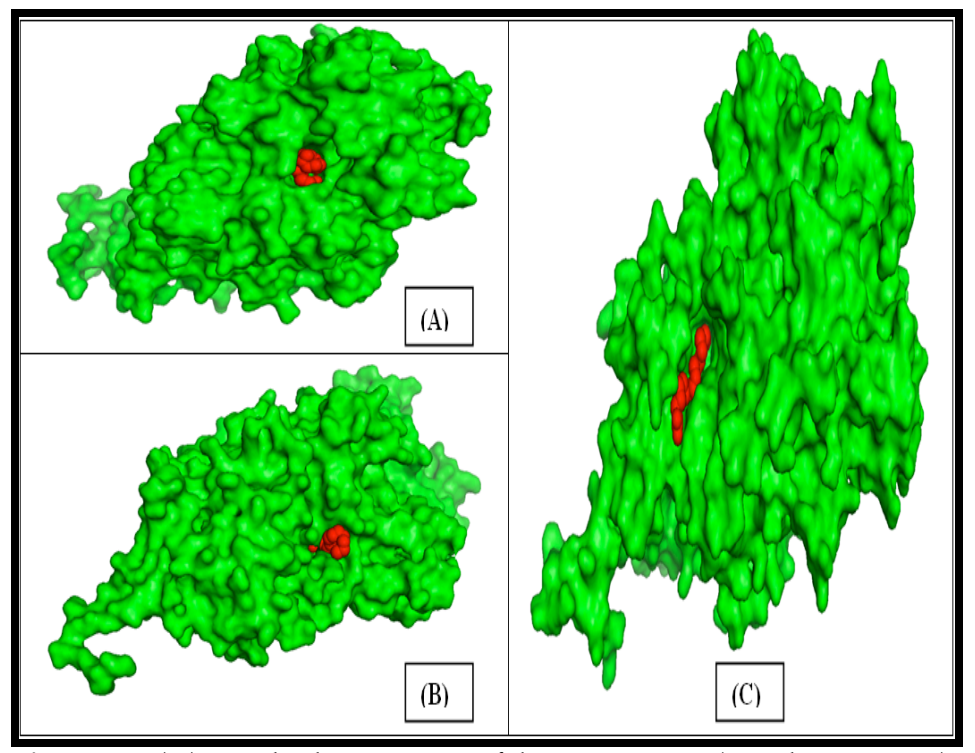

Figure 8: (A) Docked structure of lipoxygenase (Sorghum-green) with linoleate compound (red); (B) lipoxygenase (Rice-green) with linoleate compound (red); (C) lipoxygenase (Finger millet-green) with linoleate compound (red).
These domains found in a variety of membrane or lipid associated proteins. This protein has the high percentage of helix forming residues like leucine (L), alanine (A) and glutamate (E). Disulfide bonds play an important role in folding and stability of proteins. Cysteine residues were found at 239, 525, 717 position but no disulphide bond linkages as specified by CYS_REC result (Table 2). In the absence of disulphide bonds hydrogen bonding might provide stability to lipoxygenase protein. The 3D structure of sorghum and rice were also aligned to find the deviation and structural similarity using UCSF Chimera011.doc [33]. Result of structure comparison suggests that LOX-1 sorghum is highly similar with LOX-1 finger millet followed by rice and soybean on the basis of RMSD analysis. 0.263 $\AA$ RMSD value were found in between LOX-1 finger millet and sorghum with $31.47 \%$ identity, $0.298 \AA$ with $62.96 \%$ identity (between finger millet and rice), 0.391 $\AA$ (between rice and 1IK3 template structure) and $0.496 \AA$ (between finger millet and 1IK3 template structure) respectively. Furthermore, result indicated by superimposition analysis, the structure of sorghum and finger millet LOX-1 is more similar in comparison to rice and soybean template structure as well as having less deviation as shown in Figures 5 A, B, C \& D and 6. These results of structure-structure alignment revealed that finger millet is highly structurally similar with rice.

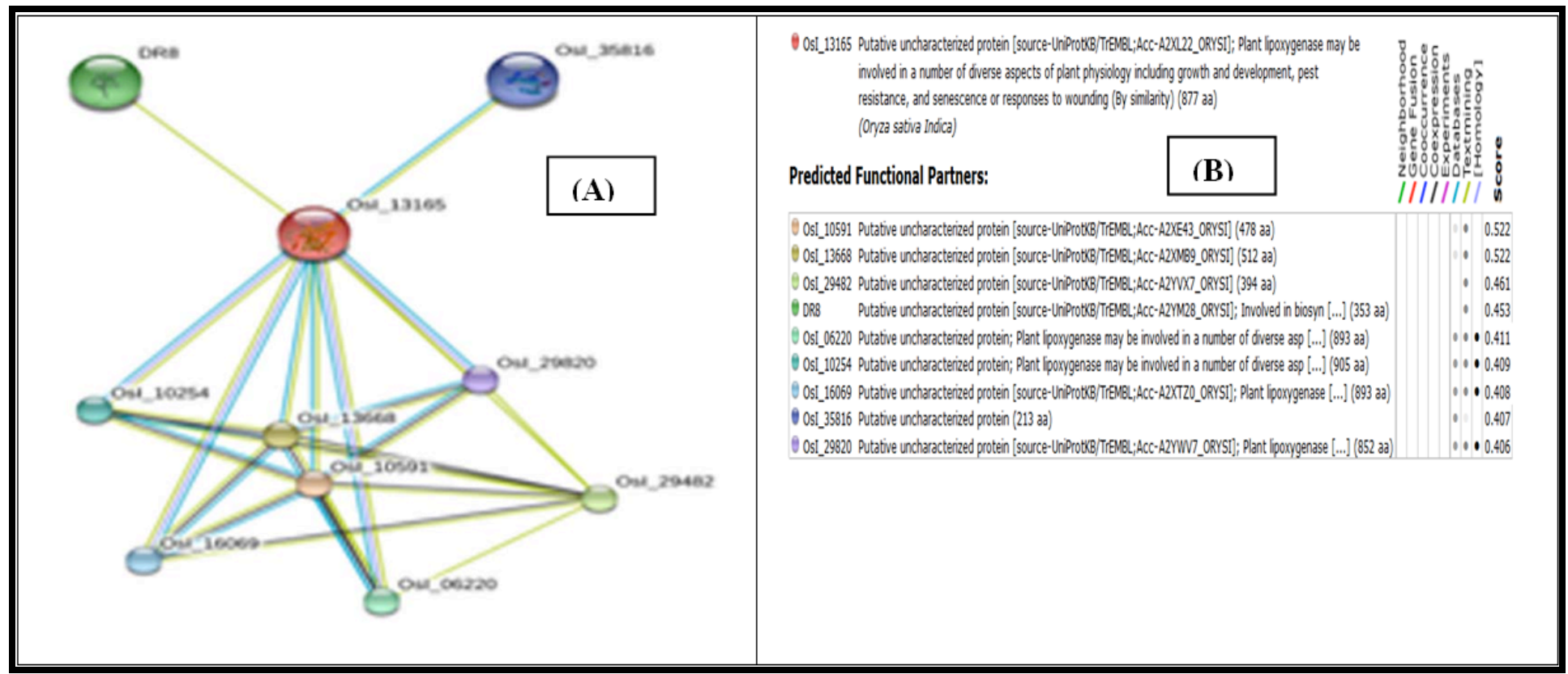

Figure 9: Protein-protein interaction network visualization. The color saturation of the edges represents the confidence score of a functional association (A) Protein-Protein interaction network with medium confidence; (B) Tabular representation of predicted interacting functional partners of LOX-1 protein. 


\section{Open access}

Table 1: Sequence alignment between query LOX from Eleusine coracana and template LOX-1 sequences with known X-ray structures from Sorghum bicolor (PDB code: LOX1), Glycine max, (PDB code: 1IK3) and (PDB code: 1ROV) Glycine max.

\begin{tabular}{cclcc}
\hline $\begin{array}{c}\text { "Source Sequence" } \\
\text { to Query source }\end{array}$ & Length & $\begin{array}{c}\text { "Target Sequence" to } \\
\text { Template source with } \\
\text { PDB code }\end{array}$ & $\begin{array}{c}\text { Length } \\
\text { "sequence } \\
\text { similarity } \\
\text { (\%)" }\end{array}$ \\
\hline LOX Eleusine coarcana & 887 & LOX-1 Sorghum bicolor & 877 & $64.42 \%$ \\
LOX Eleusine coarcana & 887 & Glycine max (1IK3) & 857 & $54.03 \%$ \\
LOX Eleusine coarcana & 887 & LOX-1 Glycine max & 839 & $53.87 \%$ \\
LOX Eleusine coarcana & 887 & Glycine max (1ROV) & 857 & $53.79 \%$ \\
\hline
\end{tabular}

Table 2: Predicted disulphide bonds (C-C) in LOX models using CYS_REC.

\begin{tabular}{cccc}
\hline Residue & Position & Bond formation & Score \\
\hline Cysteine & 239 & Not bound & -1.5 \\
Cysteine & 525 & Not bound & -27.3 \\
Cysteine & 717 & Not bound & -27.3 \\
\hline
\end{tabular}

Table 3: Predicted active sites/cavities in LOX1 Eleusine coracana, Sorghum bicolor and Oryza sativa with their positions, area and volume.

\begin{tabular}{|c|c|c|c|}
\hline $\begin{array}{c}\text { Modeled } \\
\text { Protein }\end{array}$ & $\begin{array}{c}\text { Area of } \\
\text { cavity }\end{array}$ & $\begin{array}{l}\text { Volume } \\
\text { of cavity }\end{array}$ & active-site residues \\
\hline $\begin{array}{l}\text { LOX1 E. } \\
\text { coracana }\end{array}$ & 2205.2 & 3122.5 & 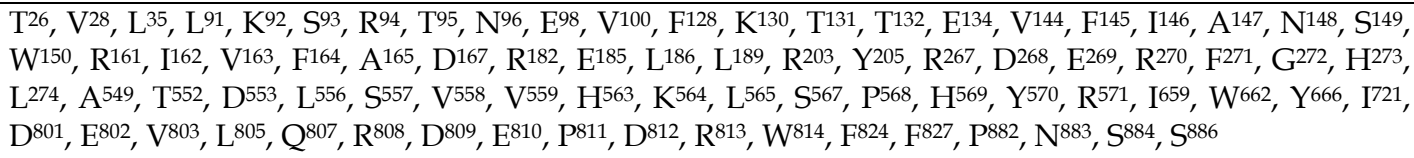 \\
\hline $\begin{array}{l}\text { LOX1 S. } \\
\text { bicolor }\end{array}$ & 2027.7 & 2753.9 & 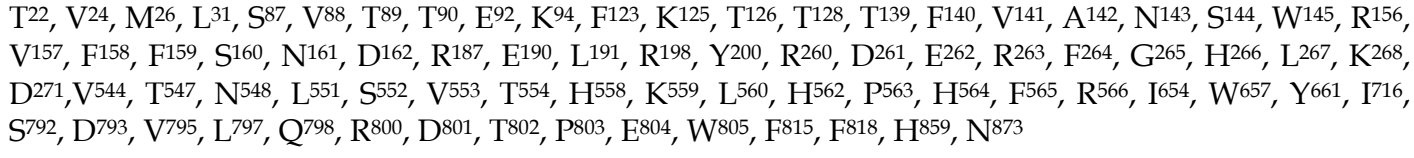 \\
\hline $\begin{array}{c}\text { LOX1 O. } \\
\text { sativa }\end{array}$ & 3198.1 & 3813.6 & $\begin{array}{l}\mathrm{M}^{24}, \mathrm{R}^{26}, \mathrm{~L}^{29}, \mathrm{D}^{30}, \mathrm{P}^{31}, \mathrm{~S}^{87}, \mathrm{Q}^{88}, \mathrm{E}^{90}, \mathrm{~K}^{119}, \mathrm{~F}^{121}, \mathrm{~K}^{123}, \mathrm{~F}^{138}, \mathrm{~V}^{139}, \mathrm{~A}^{140}, \mathrm{~N}^{141}, \mathrm{~S}^{142}, \mathrm{~W}^{143}, \mathrm{Y}^{145}, \mathrm{R}^{154}, \mathrm{I}^{155}, \mathrm{~F}^{156}, \mathrm{~F}^{157}, \\
\mathrm{~A}^{158}, \mathrm{~N}^{159}, \mathrm{R}^{179}, \mathrm{E}^{182}, \mathrm{~L}^{183}, \mathrm{~L}^{186}, \mathrm{Y}^{195}, \mathrm{Q}^{196}, \mathrm{E}^{197}, \mathrm{R}^{200}, \mathrm{Y}^{202}, \mathrm{~T}^{236}, \mathrm{G}^{237}, \mathrm{R}^{238}, \mathrm{~L}^{251}, \mathrm{E}^{255}, \mathrm{Q}^{256}, \mathrm{I}^{257}, \mathrm{Y}^{258}, \mathrm{~V}^{259}, \\
\mathrm{P}^{260}, \mathrm{~S}^{261}, \mathrm{D}^{262}, \mathrm{E}^{263}, \mathrm{R}^{264}, \mathrm{~F}^{265}, \mathrm{~A}^{266}, \mathrm{H}^{267}, \mathrm{~L}^{268}, \mathrm{~S}^{281}, \mathrm{D}^{282}, \mathrm{~F}^{283}, \mathrm{~A}^{284}, \mathrm{G}^{285}, \mathrm{Y}^{287}, \mathrm{~S}^{288}, \mathrm{~K}^{289}, \mathrm{~L}^{333}, \mathrm{D}^{336}, \mathrm{~L}^{337}, \\
\mathrm{~V}^{534}, \mathrm{~T}^{537}, \mathrm{~N}^{538}, \mathrm{~L}^{541}, \mathrm{~S}^{542}, \mathrm{~V}^{543}, \mathrm{~T}^{544}, \mathrm{Y}^{548}, \mathrm{~K}^{549}, \mathrm{~L}^{550}, \mathrm{Q}^{552}, \mathrm{P}^{553}, \mathrm{H}^{554}, \mathrm{Y}^{555}, \mathrm{R}^{556}, \mathrm{D}^{557}, \mathrm{~T}^{560}, \mathrm{I}^{561}, \mathrm{~A}^{563}, \mathrm{~L}^{564}, \\
\mathrm{Q}^{567}, \mathrm{I}^{644}, \mathrm{~W}^{647}, \mathrm{~V}^{648}, \mathrm{I}^{706}, \mathrm{~A}^{709}, \mathrm{~S}^{710}, \mathrm{H}^{713}, \mathrm{~A}^{714}, \mathrm{~F}^{718}, \mathrm{I}^{776}, \mathrm{~K}^{779}, \mathrm{H}^{780}, \mathrm{~S}^{781}, \mathrm{D}^{783}, \mathrm{E}^{784}, \mathrm{I}^{785}, \mathrm{~L}^{787}, \mathrm{Q}^{789}, \mathrm{R}^{790}, \\
\mathrm{D}^{791}, \mathrm{~T}^{792}, \mathrm{P}^{793}, \mathrm{~W}^{795}, \mathrm{~F}^{805}, \mathrm{~F}^{808}, \mathrm{~A}^{853}, \mathrm{E}^{854}, \mathrm{G}^{855}, \mathrm{P}^{862}, \mathrm{~N}^{863}, \mathrm{~S}^{864}, \mathrm{~S}^{866}, \mathrm{I}^{867}\end{array}$ \\
\hline
\end{tabular}

\section{Modeled protein submission in PMDB}

The modeled structure of LOX protein of Eleusine coracana was successfully submitted in Protein Model Database (PMDB). The PMDB ID for the submitted structure is [PM0079607]. It is publicly available for scientific community for further analysis.

\section{Analysis of Active site}

Cavities of LOX1 finger millet, sorghum and rice were predicted by CASTp and major variation (Figures 7 A \& B) among these structures was found on the basis of active site residues as reported in Table 3. Each residue was found in different position in all the structures. The result states that the active site of LOX1 finger millet might be very specific for the recognition of any insect/pest pathogenic substrates $[34,35]$.

\section{Molecular docking study of lipoxygenase with linoleate}

Molecular docking of lipoxygenase finger millet, rice and sorghum with synthetic compound i.e., linoleate were carried out using AutoDock Vina [36]. The cavity of largest volume was selected for molecular docking studies. For each protein, out of 10 different conformations of the ligand, best energetic conformation possessing the highest binding free energy score and relatively good hydrogen bond interaction was chosen. Docked Lipoxygenase of finger millet with linoleate, showing binding energy $-6.3 \mathrm{Kcal} / \mathrm{mol}$, lipoxygenase of rice showing binding energy $-5.3 \mathrm{Kcal} / \mathrm{mol}$, lipoxygenase of sorghum showing binding energy $-4.9 \mathrm{Kcal} / \mathrm{mol}$ (Figures 8 A, B \& C).

\section{Protein association network analysis}

Protein-protein interactions occur when two or multiple proteins bind together to participate in metabolic pathways and carry out their physiological functions. These interactions are important for almost every biological process in a living cell. These interactions 


\section{Open access}

help in understanding the systems biology at structural and functional level and provide the essential knowledge for the purpose of therapeutic drug targets.

In this study, LOX-1 (AHH24969.2) protein was used to find out the protein-protein functional association network. It was found that LOX-1 has 9 major association networks with other protein families which also involved in physiological activities such as pest resistance, senescence or response to wound, growth and development (Figures 9 A \& B) [37].

\section{Conclusion:}

The molecular cloning of full-length LOX-1 gene is important in plant defense against pathogen infection. Thus, an understanding of cereal specific LOX-1 using structure based function analysis is critical. Hence, we report the information gleaned using molecular docking models of linoleate with lipoxygenase from finger millet, rice and sorghum. The features of docked models showed that finger millet have higher pathogen resistance in comparison to other cereal crops.

\section{Acknowledgement:}

The authors are grateful to the Sub DIC Bioinformatics, Department of Molecular Biology \& Genetic Engineering, CBSH, G. B. Pant University of Agriculture \& Technology, Pantnagar, India for providing facilities and support to complete research work.

\section{Conflict of Interest:}

The authors declare that they have no conflict of interest.

\section{References:}

[1] Roy S et al. Bioinformation 2011 6(8): 315. [PMID: 21769194]

[2] Dinakara et al. IJPAES2014 4(3): 644.

[3] Chen H et al. Plant Physiol. 2007 143(4): 1954. [PMID: 17416643]

[4] Howe GA, Jander G Annu Rev Plant Biol. 2008 59: 41. [PMID: 18031220]

[5] Felton GW et al. J Chem Ecol. 1994 20(3): 651. [PMID: 22902801]

[6] Koch E et al. Plant Physiol. 1992 99(2): 571. [PMID: 16668924]

[7] Robert A et al., Annu.Rev. Plant Physiol. Plant Mol. Biol. 1997 48: 355. [PMID: 15012267]

[8] Avashthi $\mathrm{H}$ et al. International Journal of Computational Bioinformatics and In Silico Modeling. 2014 3(4): 454.

[9] Feussner I, Wasternack C Annu Rev Plant Biol. 2002 53: 275. [PMID: 12221977]
[10] Boyington JC et al. Science. 1993 260(5113): 1482. [PMID: 8502991]

[11] Skrzypczak-Jankun E, et al. Proteins. 1997 29(1): 15. [PMID: 9294864]

[12] Murphy JE et al. Biochem J. 2006 393(1): 107. [PMID: 16146427]

[13] Ohki I et al. Structure. 2005 13(6): 905. [PMID: 15939022]

[14] Laskowski RA et al. J. Appl. Cryst. 1993 26: 283.

[15] http://www.ncbi.nlm.nih.gov/.

[16] http://www.salilab.org/modeller.

[17] http://nihserver.mbi.ucla.edu/SAVES/.

[18] Ramachandran GN et al. J Mol Biol. 1963 7: 95. [PMID: 13990617]

[19] Eisenberg D et al. Methods Enzymol. 1997 277: 396. [PMID: 9379925]

[20] Colovos C, Yeates TO Protein Sci. 1993 2(9): 1511. [PMID: 8401235]

[21] Gill SC, Von Hippel PH Anal Biochem. 1989 182(2): 319. [PMID: 2610349]

[22] http:// www.pymol.org/.

[23] https://www.cgl.ucsf.edu/chimera/.

[24] http://cello.life.nctu.edu.tw/.

[25] Kyte J, Doolittle RF. J Mol Biol. 1982 157(1): 105. [PMID: 7108955]

[26] Castrignano T et al. Nucleic Acids Res. 2006 34: D306. [PMID: 16381873]

[27] Garg et al. Bioinformation 2016 12(2): 74.

[28] http://linux1.softberry.com/berry.phtml?topic=cys_rec\&gro up = programs\&subgroup $=$ propt.

[29] Dundas J et al. Nucleic Acids Res. 2006 34: W116. [PMID: 16844972]

[30] http://vina.scripps.edu/.

[31] Fischer AM et al. Plant J. 1999 19(5): 543. [PMID: 10504576]

[32] Hyun TK et al. PLoS One. 2014 9(11): e112946. [PMID: 25396746]

[33] https://www.cgl.ucsf.edu/chimera/.

[34] Kotapati KV et al. International Journal of Plant, Animal and Environmental Sciences. 2014 4(3): 644.

[35] Kolomiets MV et al. Plant Physiol. 2000 124(3): 1121. [PMID: 11080289]

[36] http://vina.scripps.edu/.

[37] Szklarczyk D et al. Nucleic Acids Res. 43: D447. [PMID: 25352553]

Citation: Tiwari et al. Bioinformation 12(3): 156-164 (2016)

License statement: This is an Open Access article which permits unrestricted use, distribution, and reproduction in any medium provided the original work is properly credited. This is distributed under the terms of the Creative Commons Attribution License

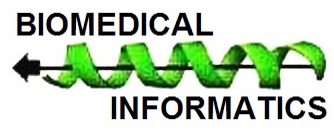

ISSN 0973-2063 (online) 0973-8894 (print)

Bioinformation 12(3): 156-164 (2016) 